\title{
CORRIGENDUM
}

\section{Peptidoglycan-degrading Enzymes in Ether-treated Cells of Neisseria gonorrhoeae}

By STEPHEN J. CHAPMAN AND HAROLD R. PERKINS

Journal of General Microbiology (1983), 129, 877-883

p. 882 , line 6

for 'only the slower moving' read 'only the faster moving' 